\title{
A Mathematical Analysis on Daily Inventory Clearance Pricing with Consumer's Reference Price
}

\author{
Takeshi Koide* \\ Department of Intelligence and Informatics, Konan University \\ Hiroaki Sandoh \\ Graduate School of Economics, Osaka University
}

(Received: December 5, 2011 / Revised: February 17, 2012 / Accepted: February 18, 2012)

\begin{abstract}
This paper discusses a clearance pricing on daily perishable products considering a reference price of consumers. The daily perishable products are sometimes sold at a discount price before closing time to stimulate demand when the number of unsold products is more than initially envisioned. The discount pricing results both in an increase of the revenue of the day and in a decrease of the disposal cost. The discounting, however, also declines a reference price of consumers which is a mental price and serves as an anchor price to judge if a current sales price is loss or gain for the consumers. An excess discounting decreases the demand for the products sold at a regular price in the future and diminishes long-term profit.

This study conducts a mathematical analysis on the clearance pricing problem for a single period with stochastic variations both on demand and on the inventory level at clearance time. The expected profit function especially depends on the response of consumers to the clearing price against their reference prices. A procedure is proposed to derive an optimal clearance price when consumers are loss-neutral. A sufficient condition is shown to obtain an optimal price for loss-averse and loss-seeking consumers by an analogous procedure.
\end{abstract}

Keywords: Optimal Pricing, Clearance, Inventory, Reference Price

* Corresponding Author, E-mail: koide@konan-u.ac.jp

\section{INTRODUCTION}

Many retail stores nowadays sell a range of prepared daily food items such as fried meals, cooked food, salad, and sushi. If such daily items are unsold at closing time, they are disposed or reused as ingredients for other items. It is a typical newsvendor problem to find an optimal amount of their initial supply to maximize an expected profit of the day.

Under practical circumstances, it is difficult to estimate demand function for target items accurately. When forecasted demand is more than actual one, retail stores often mark down a sales price to stimulate demand and to reduce the number of unsold items. If the discount pricing is conducted appropriately, it improves the profit of the day. The retail stores decrease disposal cost and might increase the revenue.

The retail stores, however, have to pay attention to the influence of the discount sales on consumers. Consumers' reference price will be declined when they purchase the products at a discount price. The reference price is a mental price and plays a role as a criterion to recognize whether an asking price is gain or loss. The reduced reference price decreases forthcoming demand 
sold at a regular price. As a result, a discount sale one day reduces profit in the future. From a viewpoint of profitability, retail stores should determine the clearing discount price carefully with considering its influence on long-term profit.

The reference price is initially proposed as a reference point in the prospect theory by Kahneman and Tversky (1979). The reference price itself is actively researched especially in the area of marketing science. Kalyanaram and Winner (1995) summarized past research with respect to the reference price and mentioned that the reference price is generated by a series of past sales prices. Consumers react differently according to whether the sales price is less or greater than the reference prices of the consumers. The asymmetric reaction is a key concept on behavioral economics well-researched recently.

Our study aims to derive an optimal clearance pricing on daily perishable products to maximize a longterm expected profit. This paper focuses on a single period model as a first step for the long-term optimization. A model is proposed where stochastic demand, stochastic inventory level, which means the amount of unsold items, and consumers' reference price effect are considered. Greenleaf (1995) proposed a model for the first time to derive an optimal pricing considering the reference price effect and Kopalle et al. (1996) extended his model. Their models, however, are for promotion planning and do not contain the concept of inventory level. Popescu and $\mathrm{Wu}$ (2007) showed optimal pricing policies for the promotion problem with more general type of demand function, but inventory level is also excluded from their models. The above studies treat deterministic demand functions and derive an optimal price through the dynamic programming.

The problem to determine an optimal supply quantity toward uncertain demand is well-known as newsvendor problem. The newsvendor problem is originally studied by Arrow et al. (1951) and various models have been proposed since then. Petruzzi and Dada (1999) discussed the relationship between pricing and inventory control. They summarized past research with respect to pricing and the newsvendor problem. They introduced a model which treats stochastic demand and explores both an optimal price and an optimal inventory level, but the reference price effect is not discussed in the model.

In this study, an expected profit function is formulated and analyzed mathematically. First, a profit function with deterministic demand and inventory level in a single period model is discussed. The result reveals that the shape of the profit function depends on the consumer's attitude toward gain and loss, in other words, on whether consumers are loss-neutral (LN), loss-averse (LA), or loss-seeking (LS). Then, the discussed model is extended to treat stochastic demand and inventory level and a sufficient condition is shown to obtain a unique optimal price to maximize an expected profit.

\section{PROBLEM DESCRIPTION}

Consider a firm deals in a type of product under monopoly. The target time horizon is limited to a single period in this study. The firm prepares a certain amount of the products before opening time at a unit procurement $\operatorname{cost} c(>0)$ and starts to sell the product at a regular price $p_{H}$. No replenishment is considered in this model. After closing time, unsold products are salvaged or disposed at a unit cost $h$, which means that the unsold products are salvaged if $h<0$ and they are disposed otherwise. Let $s(>0)$ be a unit penalty cost for an opportunity loss.

At a prescheduled time during the operating hours, the firm can discount the products to stimulate demand. This study focuses on the optimal discount pricing. The only decision variable in this model is the discount price $p$ in the range $\left[p_{L}, p_{H}\right]$. The firm determines the price $p$ before the prescheduled time in advance with considering uncertain inventory level $Q$ and supposed consumer's reference price $r$ for the products. If some products are unsold at the prescheduled time, the unsold products are sold at price $p$ from then to closing time. The reference price $r$ exists in the range $\left[p_{L}, p_{H}\right]$. The inventory level $Q$ is assumed to be a random variable and it is given by $Q=q+\varepsilon_{q}$, where $q$ is the average of $Q$ and $\varepsilon_{q}$ is a random factor whose mean is 0 and range is $\left[q_{L}, q_{H}\right]$.

The demand function for the product $D(p, r)$ includes the reference price effect and stochastic variation:

$$
\begin{aligned}
& D(p, r)=d(p, r)+\varepsilon_{d}, \\
& d(p, r)= \begin{cases}d_{G}(p, r) \text { if } p<r \\
d_{L}(p, r) & \text { otherwise, }\end{cases} \\
& d_{G}(p, r)=\beta_{0}-\beta_{1} p+\beta_{2 G}(r-p) \equiv B_{0 G}(r)-B_{1 G} p, \\
& d_{L}(p, r)=\beta_{0}-\beta_{1} p+\beta_{2 L}(r-p) \equiv B_{0 L}(r)-B_{1 L} p .
\end{aligned}
$$

The positive parameters $\beta_{2 G}$ and $\beta_{2 L}$ respectively represent the degree of the reference price effect when consumers recognize the discount price $p$ as a gain $(p<$ $r)$ and a loss $(p>r)$. The parameters characterize the consumer's response toward the selling price. The consumers with $\beta_{2 G}<\beta_{2 L}, \beta_{2 G}=\beta_{2 L}$, and $\beta_{2 G}>\beta_{2 L}$ are called $\mathrm{LA}, \mathrm{LN}$, and LS, respectively. All of the parameters $B_{0 G}(r), B_{0 L}(r), B_{1 G}, B_{1 L}$ are consequently positive. The random factor $\varepsilon_{d}$ in the demand function, whose mean is 0 and range is $\left[d_{L}, d_{H}\right]$, is assumed to be independent of both the sales price $p$ and reference price $r$. Assume $-h$ $<c<p_{L}$ and $D(p, r)>0$ for any $p, r \in\left[p_{L}, p_{H}\right]$.

\section{OPTIMAL PRICING IN A DETERMINIS- TIC CONDITION}

This section discusses the optimal pricing in case that the demand and inventory level are deterministic as a simple case. In other words, we here treat the case where 


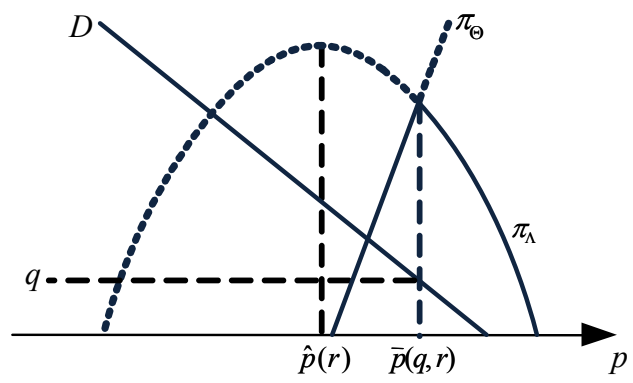

(a) $\hat{p}(r) \leq \bar{p}(q, r)$

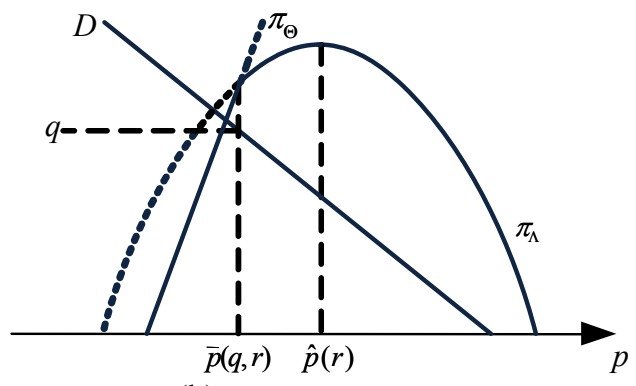

(b) $\hat{p}(r)>\bar{p}(q, r)$

Figure 1. $\pi(p, q, r)$ for LN Consumers.

$\varepsilon_{d}$ and $\varepsilon_{q}$ are constantly equal to 0 .

\subsection{Optimal Pricing for Loss-Neutral Consumers}

This subsection confines our discussion to the optimal pricing for LN consumers. Let $\beta_{2 G}=\beta_{2 L}=\beta_{2}$ then the demand function $D(p, r)=d(p, r)$ is given by the following equation:

$$
d(p, r)=\beta_{0}-\beta_{1} p+\beta_{2}(r-p) \equiv B_{0}(r)-B_{1} p .
$$

The both parameters $B_{0}(r)$ and $B_{1}$ are positive. Let $\bar{p}(q$, $r$ ) be the price $p$ which satisfies $d(p, r)=q$. Then, it holds

$$
\bar{p}(q, r)=\frac{B_{0}(r)-q}{B_{1}} .
$$

When the products are sold at price $p$ for lossneutral consumers with reference price $r$ and the inventory level $Q$ is a constant $q$, the profit $\pi(p, q, r)$ is represented by

$$
\begin{aligned}
& \pi(p, q, r)= \begin{cases}\pi_{\Theta}(p, q, r) & \text { if } p<\bar{p}(q, r) \\
\pi_{\Lambda}(p, q, r) & \text { otherwise, }\end{cases} \\
& \pi_{\Theta}(p, q, r)=p q-c q-s[D(p, r)-q], \\
& \pi_{\Lambda}(p, q, r)=p D(p, r)-c q-h[q-D(p, r)] .
\end{aligned}
$$

Let $\hat{p}(r)$ be the price $p$ which maximizes $\pi_{\Lambda}(p, q, r)$. A simple analysis of $\pi_{\Lambda}(p, q, r)$ derives

$$
\hat{p}(r)=\frac{1}{2}\left(\frac{B_{0}(r)}{B_{1}}-h\right) .
$$

Let $p^{*}(q, r)$ be the price to maximize $\pi(p, q, r)$, then the following theorem is proved.

Theorem 1: When both the demand function $D(p, r)$ and the inventory level $Q$ are deterministic and consumers are $L N$, the optimal price $p^{*}(q, r)$ which maximizes the profit $\pi(p, q, r)$ is derived by the following equation:

$$
p^{*}(q, r)=\min \left[\max \left\{\hat{p}(r), \bar{p}(q, r), p_{L}\right\}, p_{H}\right] .
$$

Proof: The function $\pi_{\Theta}(p, q, r)$ monotonically increases since

$$
\frac{\partial \pi_{\Theta}(p, q, r)}{\partial p}=q-s \frac{\partial D(p, r)}{\partial p}=q+s B_{1}>0 .
$$

Meanwhile, the function $\pi_{\Lambda}(p, q, r)$ is a concave function shown as follows:

$$
\begin{aligned}
& \frac{\partial \pi_{\Lambda}(p, q, r)}{\partial p}=B_{0}(r)-B_{1}(2 p+h), \\
& \frac{\partial^{2} \pi_{\Lambda}(p, q, r)}{\partial p^{2}}=-2 B_{1}<0 .
\end{aligned}
$$

Hence, the profit $\pi(p, q, r)$ increases monotonically in the range of $p<\bar{p}(q, r)$, where $\pi(p, q, r)=\pi_{\Theta}(p, q, r)$, and it is concave in the range of $p \geq \bar{p}(q, r)$, where $\pi(p, q$, $r)=\pi_{\Lambda}(p, q, r)$. Figure 1 depicts the shape of $\pi(p, q, r)$. When the two prices $\bar{p}(q, r)$ and $\bar{p}(r)$, exits in $\left[p_{L}, p_{H}\right]$, the profit function $\pi(p, q, r)$ monotonically increases for $p \leq \max \{\bar{p}(q, r), \hat{p}(r)\}$. and monotonically decreases for $p$ $>\max \{\bar{p}(q, r), \hat{p}(r)\}$. Then, the greater between $\bar{p}(q, r)$ and $\hat{p}(r)$ maximizes the profit $\pi(p, q, r)$.

Corollary 1: The optimal price $p^{*}(q, r)$ by Equation (11) is expressed as follows:

$p^{*}(q, r)= \begin{cases}\hat{p} & \text { if } \frac{1}{2} d(-h, r) \leq q, d\left(2 p_{H}+h, r\right) \leq 0 \leq d\left(2 p_{L}+h, r\right), \\ \bar{p} & \text { if } \frac{1}{2} d(-h, r)>q, d\left(p_{H}, r\right) \leq q \leq d\left(p_{L}, r\right), \\ p_{L} & \text { if } d\left(p_{L}, r\right)<q, d\left(2 p_{L}+h, r\right)>0, \\ p_{H} & \text { otherwise. }\end{cases}$

Proof:

$$
\begin{aligned}
& \hat{p}(r) \underset{>}{>} p \Leftrightarrow d(2 p+h, r) \frac{<}{>}, \\
& \bar{p}(q, r) \underset{>}{>} p \Leftrightarrow d(p, r) \frac{<}{>} q, \\
& \hat{p}(r) \underset{>}{>} \bar{p}(q, r) \Leftrightarrow \frac{1}{2} d(-h, r) \underset{>}{>} q,
\end{aligned}
$$

The above inequalities directly derive the corollary. 

gives

Differentiating $\bar{p}(q, r)$ and $\hat{p}(r)$ with respect to $r$

$$
\frac{\partial \bar{p}(q, r)}{\partial r}=\frac{\beta_{2}}{B_{1}}>\frac{\beta_{2}}{2 B_{1}}=\frac{\partial \hat{p}(r)}{\partial r}>0 .
$$

Hence, both $\bar{p}(q, r)$ and $\hat{p}(r)$ increase monotonically with respect to $r$ and the increment of $\bar{p}(q, r)$ is twice that of $\hat{p}(r)$. Figure 2 illustrates the region regarding the optimal price with assuming $p_{L} \leq \bar{p}(q, r) \leq p_{H}$ and $p_{L}$ $\leq \hat{p}(q, r) \leq p_{H}$. An numerical example for the optimal price $p^{*}(q, r)$ are shown as three-dimensional images in Figure 3 under the following parameters setting: $\beta_{0}=$ $100, \beta_{1}=0.1, \beta_{2}=0.01$, and $h=50$.

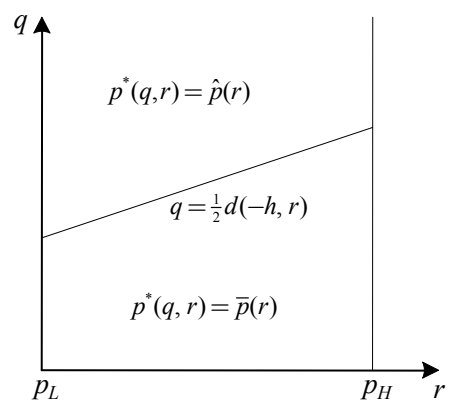

Figure 2. Regions for Optimal Price.

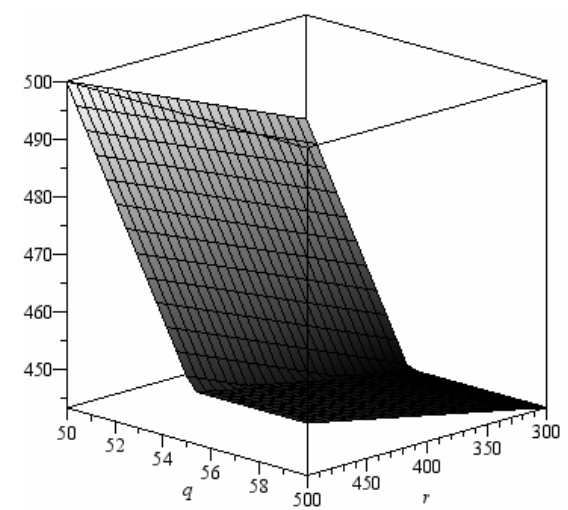

Figure 3. Optimal Prices in 3D Image.

Here, let $\bar{\beta}_{2}$ be the $\beta_{2}$ which satisfies $\hat{p}(r)=r$, then Equation (10) yields

$$
\bar{\beta}_{2}=\frac{\beta_{0}-\beta_{0}(2 r+h)}{r+h}
$$

Lemma 1 explains the influence of $\beta_{2}$ on $\pi_{\Lambda}(p, q, r)$ and $\hat{p}(r)$.

Lemma 1: The price $\hat{p}(r)$ is decreasing with respect to $\beta_{2}$. The profit $\pi_{\Lambda}(p, q, r)$ is increasing, constant, and decreasing with respect to $\beta_{2}$ for $p<r, p=r$, and $p>r$, respectively. Furthermore, the maximum of $\pi_{\Lambda}(p, q, r)$ is decreasing and increasing with respect to $\beta_{2}$ for $\beta_{2}$ $<\bar{\beta}_{2}$ and $\beta_{2}>\bar{\beta}_{2}$, respectively.

Proof: Differentiating Equation (10) derives

$$
\frac{\partial \hat{p}(r)}{\partial \beta_{2}}=\frac{1}{2} \frac{\partial}{\partial \beta_{2}} \frac{\beta_{0}+r \beta_{2}}{\beta_{1}+\beta_{2}}=-\frac{1}{2} \frac{d(r, r)}{\left(\beta_{1}+\beta_{2}\right)^{2}}<0,
$$

hence $\hat{p}(r)$ is decreasing with respect to $\beta_{2}$. Similarly, differentiating Equation (9) derives

$$
\frac{\partial \pi_{\Lambda}(p, q, r)}{\partial \beta_{2}}=(p+h) \frac{\partial D(p, r)}{\partial \beta_{2}}=(p+h)(r-p) .
$$

From the assumption that $p+h>0$, Equation (22) proves the property on $\pi_{\Lambda}(p, q, r)$. Finally, differentiating $\pi_{\Lambda}(\hat{p}$ $(r), q, r)$ with respect to $\beta_{2}$ gives

$$
\frac{\partial \pi_{\Lambda}(\hat{p}(r), q, r)}{\partial \beta_{2}}=\frac{\partial \pi_{\Lambda}(\hat{p}(r), q, r)}{\partial \hat{p}(r)} \frac{\partial \hat{p}(r)}{\partial \beta_{2}} .
$$

Equations (21), (22), and (23) prove the last property regarding the maximum of profit $\pi_{\Lambda}(\hat{p}(r), q, r)$.

The profit functions $\pi_{\Lambda}(p, q, r)$ with several values of $\beta_{2}$ are depicted in Figure 4 , where $r=450$ and $\bar{\beta}=0.02$. Since $\hat{p}(r)$ decreases monotonically with respect to $\beta_{2}$, the maximum vertex of $\pi_{\Lambda}(p, q, r)$ moves leftward and downward and approaches toward the point $\left(r, \pi_{\Lambda}(r, q\right.$, $r)$ ) with increasing $\beta_{2}$ to $\bar{\beta}_{2}$. The vertex of $\pi_{\Lambda}(p, q, r)$ moves leftward and upward from $\left(r, \pi_{\Lambda}(r, q, r)\right)$ when $\beta_{2}$ increases from $\beta_{2}=\bar{\beta}_{2}$.

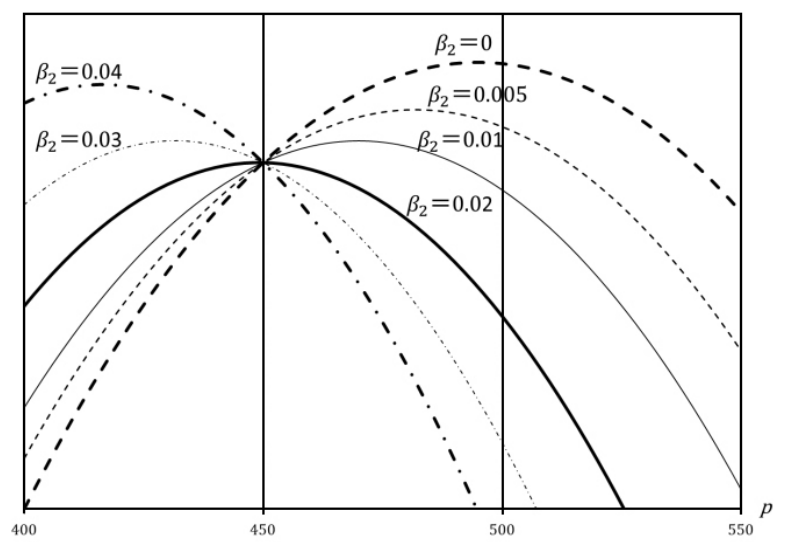

Figure 4. $\pi_{\Lambda}(p, q, r)$ with Several Values of $\beta_{2}$ on where $r=450$.

\subsection{Optimal Pricing for Asymmetry Consumers}

This subsection discusses the optimal pricing for LA and LS consumers, namely when it holds that $\beta_{2 G}$ $\neq \beta_{2 L}$. In this case, the expected demand function $d(p, r)$ can be expressed as follows:

$$
d(p, r)= \begin{cases}\min \left\{d_{G}(p, r), d_{L}(p, r)\right\}, & \text { for } L A \\ \max \left\{d_{G}(p, r), d_{L}(p, r)\right\}, & \text { for } L S\end{cases}
$$


since both $d_{G}(p, r)$ and $d_{L}(p, r)$ linear functions with respect to $p$ and they have a common point at $p=r$.

In the same manner in the previous subsection, as $\bar{p}(q, r)$ was defined as follows:

$$
\bar{p}(q, r)= \begin{cases}\frac{B_{0 G}(r)-q}{B_{1 G}} \equiv \bar{p}_{G}(q, r) & \text { if } d(r, r)<q, \\ \frac{B_{0 L}(r)-q}{B_{1 L}} \equiv \bar{p}_{L}(q, r) & \text { otherwise. }\end{cases}
$$

Figure 5 indicates the two prices $\bar{p}_{G}(q, r)$ and $\bar{p}_{L}(q, r)$ Since Equation (7) also holds for LA and LS consumers, $\pi(p, q, r)=\pi_{\Theta}(p, q, r)$ for $p<\bar{p}(q, r)$. From Equation (8), the profit function $\pi_{\Theta}(p, q, r)$ in the case of inventory shortage is decreasing as $d(p, r)$ increases, and then it is expressed as

$$
\pi_{\Theta}(p, q, r)=\left\{\begin{array}{l}
\max \left\{\pi_{\Theta G}(p, q, r), \pi_{\Theta L}(p, q r)\right\}, \text { for } L A \\
\min \left\{\pi_{\Theta G}(p, q, r), \pi_{\Theta L}(p, q r)\right\}, \text { for } L S
\end{array}\right.
$$

where $\pi_{\Theta G}(p, q, r)$ and $\pi_{\Theta L}(p, q, r)$ are the $\pi_{\Theta}(p, q, r)$ in Equation (8) with $\beta_{2}=\beta_{2 G}$ and $\beta_{2}=\beta_{2 L}$, respectively. The function $\pi_{\Theta}(p, q, r)$ in Equation (26) monotonically increases with respect to $p$ since both $\pi_{\Theta G}(p, q, r)$ and $\pi_{\Theta L}(p, q, r)$ increase monotonically as proved by Equation (12). The profit $\pi(p, q, r)$ for LA and LS consumers increases monotonically in the range $p<\bar{p}(q, r)$.

The profit function $\pi_{\Lambda}(p, q, r)$ in the case of excessive inventory is expressed as

$$
\pi_{\Lambda}(p, q, r)= \begin{cases}\min \left\{\pi_{\Lambda G}(p, q, r), \pi_{\Lambda L}(p, q r)\right\}, & \text { for } L A \\ \max \left\{\pi_{\Lambda G}(p, q, r), \pi_{\Lambda L}(p, q r)\right\}, & \text { for } L S\end{cases}
$$

since Equation (9) shows $\pi_{\Lambda}(p, q, r)$ increases as $d(p, r)$ increases. The profit functions $\pi_{\Lambda G}(p, q, r)$ and $\pi_{\Lambda L}(p, q$, $r)$ are the $\pi_{\Lambda}(p, q, r)$ in Equation (9) with $\beta_{2}=\beta_{2 G}$ and $\beta_{2}$ $=\beta_{2 L}$, respectively. The two profit functions $\pi_{\Lambda \mathrm{G}}(p, q, r)$ and $\pi_{\Lambda L}(p, q, r)$ have a common point on $p=r$. Let $\hat{p}_{G}(r)$ and $\hat{p}_{L}(r)$ be respectively the prices on which $\pi_{\Lambda G}(p, q, r)$ and $\pi_{\Lambda L}(p, q, r)$ have a maximum, namely

$$
\begin{aligned}
& \hat{p}_{G}(r)=\frac{1}{2}\left(\frac{B_{0 G}(r)}{B_{1 G}}-h\right), \\
& \hat{p}_{L}(r)=\frac{1}{2}\left(\frac{B_{0 L}(r)}{B_{1 L}}-h\right) .
\end{aligned}
$$

Lemma 1 concludes that it holds $\hat{p}_{L}(r)<\hat{p}_{G}(r)$ for LA consumers and $\hat{p}_{G}(r)<\hat{p}_{L}(r)$ for LS consumers. Then, Lemma 1 restricts the possibility of the shapes of the profit functions $\pi_{\Lambda}(p, q, r)$ for LA and LS consumers, represented in Figure 6 and Figure 7, respectively. The function for LA consumers is concave in any cases in Figure 6. The function for LS consumers is also concave except in the case of $\hat{p}_{G}(r)<\hat{p}_{L}(r)$, when the function is bimodal. This discussion introduces the following theorem as a procedure to derive the optimal price for the asymmetry consumers.

Theorem 2: When both the demand function $D(p, r)$ and the inventory level $Q$ are deterministic and consumers are $L A$ or $L S$, the optimal price $p^{*}(q, r)$ which maximizes the profit $\pi(p, q, r)$ is derived by the following equations:

$$
\begin{aligned}
& p^{*}(q, r)=\arg \max _{p \in P_{2}^{*}} \pi(p, q, r), \\
& P_{1}^{*}= \begin{cases}\left\{\hat{p}_{G}(r), \hat{p}_{L}(r)\right\} & \text { if } \hat{p}_{G}(r) \leq r \leq \hat{p}_{L}(r), \\
\left\{\hat{p}_{G}(r)\right\} & \text { if } \max \left\{\hat{p}_{G}(r), \hat{p}_{L}(r)\right\} \leq r, \\
\left\{\hat{p}_{L}(r)\right\} & \text { if } \min \left\{\hat{p}_{G}(r), \hat{p}_{L}(r)\right\} \geq r, \\
\{r\} & \text { otherwise, }\end{cases} \\
& P_{2}^{*}=\left\{\min \left[\max \left\{p, \bar{p}(q, r), p_{L}\right\}, p_{H}\right] \mid p \in P_{1}^{*}\right\} .
\end{aligned}
$$

Proof: The set $P_{1}{ }^{*}$ consists of the candidate prices to maximize $\pi_{\Lambda}(p, q, r)$ without considering the lower and upper limits of the sales price. For $\hat{p}_{G}(r) \leq r \leq \hat{p}_{L}(r)$, in the middle case in Figure $7, \pi_{\Lambda}(p, q, r)$ is bimodal and both $\hat{p}_{G}(r)$ and $\hat{p}_{L}(r)$ could be optimal. In the case of $\max \left\{\hat{p}_{G}(r), \hat{p}_{L}(r)\right\} \leq r, \hat{p}_{G}(r)$ is the optimal price both for LA and LS consumers. Similarly, $\hat{p}_{L}(r)$ is the optimal

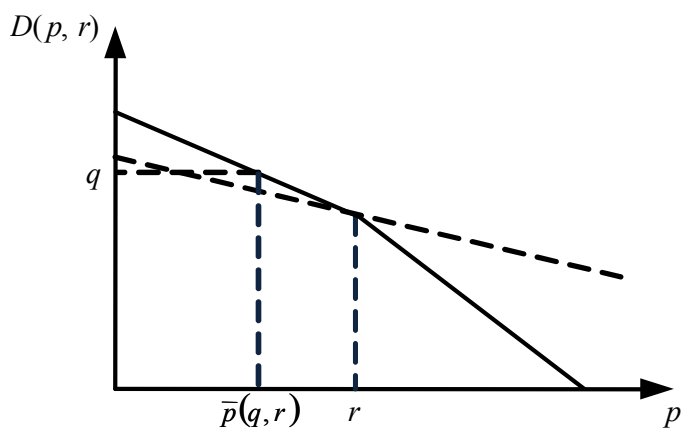

(a) $d(r, r)<q$

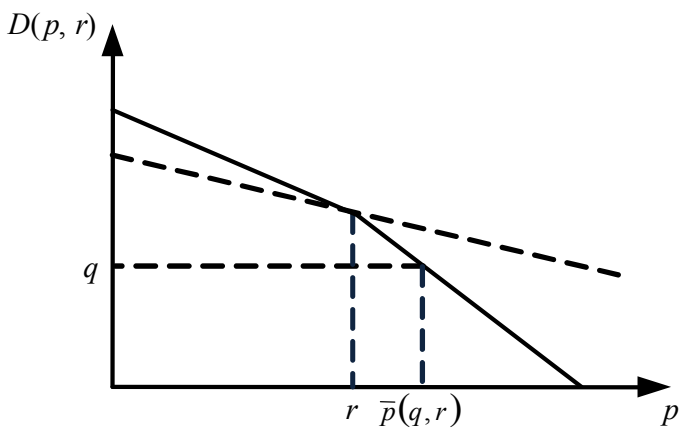

(b) $d(r, r)>q$

Figure 5. $\bar{p}(q, r)$ for LA or LS Consumers. 


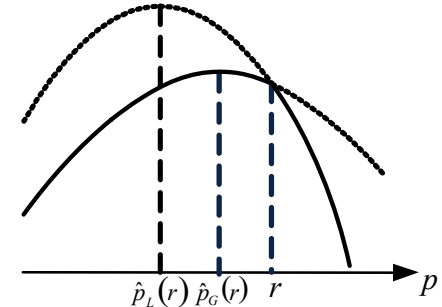

(a) $\hat{p}_{L}(r)<\hat{p}_{G}(r) \leq r$

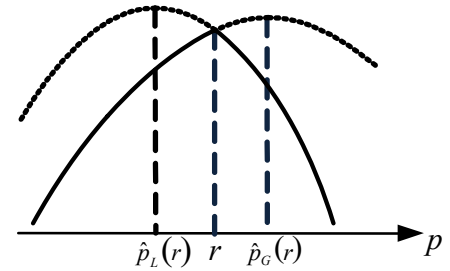

(b) $\hat{p}_{L}(r) \leq r \leq \hat{p}_{G}(r)$

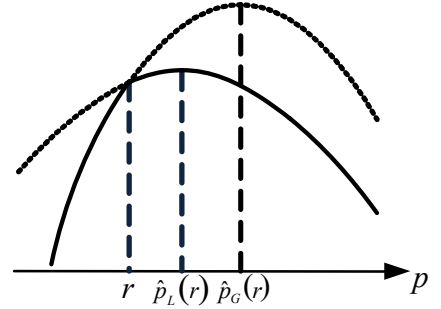

(c) $r \leq \hat{p}_{L}(r)<\hat{p}_{G}(r)$

Figure 6. $\pi_{\Lambda}(p, q, r)$ for LA Consumers.

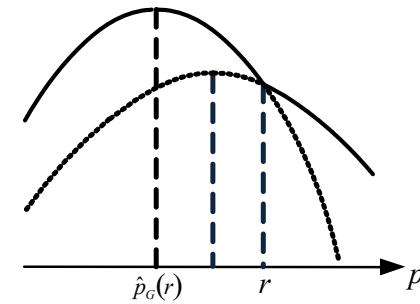

(a) $\hat{p}_{L}(r)<\hat{p}_{G}(r) \leq r$

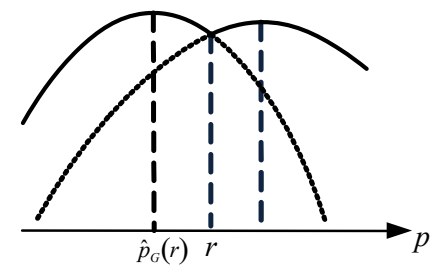

(b) $\hat{p}_{L}(r) \leq r \leq \hat{p}_{G}(r)$

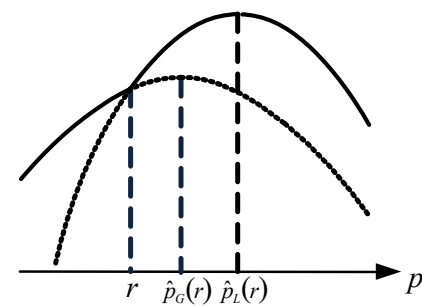

(c) $r \leq \hat{p}_{L}(r)<\hat{p}_{G}(r)$

Figure 7. $\pi_{\Lambda}(p, q, r)$ for LS Consumers.

when it holds $\min \left\{\hat{p}_{G}(r), \hat{p}_{L}(r)\right\} \geq r$. In the last case, the middle case in Figure $6, r$ is the optimal. In the same manner in Theorem 1, Equation (32) ascertains that the candidate prices in $P_{1}{ }^{*}$ to be optimal for $\pi(p, q, r)$.

Note that the cardinality of $P_{2}{ }^{*}$ is two in the case of $\hat{p}_{G}$ $(r) \leq r \leq \hat{p}_{L}(r)$. In the other cases, the cardinality is one and Equation (32) explores the optimal price without Equation (30).

\section{OPTIMAL PRICING IN STOCHASTIC CONDITION}

This section discusses the optimal pricing in case that the demand $D(p, r)$ and inventory level $Q$ are stochastic.

\subsection{Optimal Pricing for LN Consumers}

This subsection confines our discussion to the optimal pricing for LN consumers. The demand function $D(p, r)$ is defined by Equations (1) and (5). Define new variables $z=q-d(p, r)$ and $\varepsilon=\varepsilon_{q}-\varepsilon_{d}$. in accordance with Petruzzi and Dada (1999). Note that

$$
Q-D(p, r)=z+\varepsilon
$$

The average of $\varepsilon$ is 0 and the range of $\varepsilon$ is $\left[q_{L}-d_{H}\right.$, $\left.q_{H}-d_{L}\right]$. Then, the profit $\pi(p, q, r)$ is expressed

$$
\pi(p, q, r)= \begin{cases}p D(p, r)-c Q-h(z+\varepsilon) & \text { if } z+\varepsilon \geq 0 \\ p D(p, r)-c Q+(p+s)(z+\varepsilon) & \text { otherwise }\end{cases}
$$

Let $f(\cdot)$ and $F(\cdot)$ be the probabilistic density function and the distribution function of the variable $\varepsilon$. Define $\bar{F}(\cdot)=1-F(\cdot)$. The expected profit $\Pi(p, q, r)$, hence, is obtained by

$$
\begin{aligned}
\Pi(p, q, r)= & p d(p, r)-c q-h \int_{-z}^{z_{H}}(z+u) f(u) d u \\
& +(p+s) \int_{z_{L}}^{-z}(z+u) f(u) d u .
\end{aligned}
$$

The expected profit $\Pi(p, q, r)$ can be rewritten by

$$
\begin{aligned}
& \Pi(p, q, r)=\Psi(p, r)-L(p, z), \\
& \Psi(p, r)=(p-c) d(p, r), \\
& L(p, z)=(c+h) \Lambda(z)+(p-c+s) \Theta(z), \\
& \Lambda(z)=\int_{-z}^{z_{H}}(z+u) f(u) d u \\
& \Theta(z)=-\int_{z_{L}}^{-z}(z+u) f(u) d u .
\end{aligned}
$$

In Equation (36), $\Psi(p, r)$ and $L(p, z)$ respectively imply the profit for $Q=D(p, r)$ and the expected cost incurred by excess or deficiency of inventory. The expected volumes of excess and deficiency of inventory are denoted by $\Lambda(z)$ and $\Theta(z)$ defined in Equations (39) and (40), respectively. The following theorem derives the optimal price $p^{*}(q, r)$ to maximize the expected profit $\Pi(p, q, r)$.

Theorem 3: When both the demand function $D(p, r)$ and the inventory level $Q$ are stochastic and consumers are $L N$, the optimal price $p^{*}(q, r)$ which maximizes the expected profit $\Pi(p, q, r)$ is derived by the following equation: 


$$
p^{*}(q, r)=\min \left[\max \left\{\hat{p}(q, r), p_{L}\right\}, p_{H}\right]
$$

where $\hat{p}(q, r)$ is the unique solution of $g(p, q, r)=0$ :

$g(p, q, r)=B_{0}(r)-(2 p+h) B_{1}+(p+h+s) B_{1} F(-z)-\Theta(z)$.

Proof: Differentiating from Equations (36) to (40) with respect to $p$ yields

$$
\begin{aligned}
& \frac{\partial z}{\partial p}=\frac{\partial}{\partial p}\{q-d(p, r)\}=B_{1} \\
& \frac{\partial \Lambda(z)}{\partial p}=\frac{\partial}{\partial z}\left\{\int_{-z}^{z_{H}}(z+u) f(u) d u\right\} \frac{\partial z}{\partial p}=B_{1} \bar{F}(-z) \\
& \frac{\partial \Theta(z)}{\partial p}=\frac{\partial}{\partial z}\left\{-\int_{z_{L}}^{-z}(u+z) f(u) d u\right\} \frac{\partial z}{\partial p}=-B_{1} F(-z) \\
& \frac{\partial L(p, z)}{\partial p}=(c+h) B_{1}+\Theta(z)-(p+h+s) B_{1} F(-z) \\
& \frac{\partial \Psi(p, r)}{\partial p}=\frac{\partial}{\partial p}\{(p-c) d(p, r)\}=B_{0}(r)-(2 p-c) B_{1}, \\
& \frac{\partial \Pi(p, q, r)}{\partial p}=B_{0}(r)-(2 p+h) B_{1} \\
& \quad+(p+h+s) B_{1} F(-z)-\Theta(z) \\
& \frac{\partial^{2} \Pi(p, q, r)}{\partial p^{2}}=-2 B_{1} \bar{F}(-z)-(p+h+s) B_{1}^{2} f(-z)
\end{aligned}
$$

Using the assumption of $h<p_{L}$, Equations (48) and (49) prove that $\Pi(p, q, r)$ is concave with respect to $p$ and has a unique maximum $\hat{p}(q, r)$ which satisfies $g(p, q, r)=0$.

Corollary 2: The optimal price $p^{*}(q, r)$ by Equation (41) is expressed as follows:

$$
p^{*}(q, r)= \begin{cases}p_{L} & \text { if } g\left(p_{L}, q, r\right) \leq 0 \\ p_{H} & \text { if } g\left(p_{H}, q, r\right) \geq 0 \\ \hat{p} & \text { otherwise. }\end{cases}
$$

Proof: It is obviously proved from the concavity of the function $\Pi(p, q, r)$.

Similarly to the deterministic case, let $\bar{\beta}_{2}$ be the $\beta_{2}$ which satisfies $\hat{p}(r)=r$, then $\bar{\beta}_{2}$ is obtained from the equation $g(r, q, r)=0$ :

$$
\bar{\beta}_{2}=\frac{d(r, r)-\theta(q-d(r, r))}{(r+h+s) \bar{F}(d(r, r)-q)-s}-\beta_{1}
$$

provided that $(r+h+s) \bar{F}(d(r, r)-q)-s$ is not equal to 0 . The next lemma reveals a property on $\Pi(p, q, r)$ with respect to $\beta_{2}$.

Lemma 2: The expected profit $\Pi(p, q, r)$ is concave with respect to $\beta_{2}$. Furthermore, under the circumstance which satisfies the following inequality

$$
(p+h+s) \bar{F}(d(p, r)-q)>s
$$

$\Pi(p, q, r)$ has the following properties: (i) $\Pi(p, q, r)$ is increasing, constant, and decreasing with respect to $\beta_{2}$ for $p<r, p=r$, and $p>r$, respectively; (ii) The maximum of $\Pi(p, q, r)$ is decreasing and increasing with respect to $\beta_{2}$ for $\beta_{2}<\bar{\beta}_{2}$ and $\beta_{2}>\bar{\beta}_{2}$, respectively; (iii) $\hat{p}(q, r)$ is decreasing with respect to $\beta_{2}$.

Proof: Differentiating from Equations (36) to (40) with respect to $\beta_{2}$ yields

$$
\begin{aligned}
& \frac{\partial z}{\partial \beta_{2}}=\frac{\partial}{\partial \beta_{2}}\{q-d(p, r)\}=p-r \\
& \frac{\partial L(p, z)}{\partial \beta_{2}}=(c+h)(p-r)-(p+h+s)(p-r) F(-z) \\
& \frac{\partial \Psi(p, r)}{\partial \beta_{2}}=(p-c) \frac{\partial d(p, r)}{\partial \beta_{2}}=-(p-r)(p-c) \\
& \frac{\partial \Pi(p, q, r)}{\partial \beta_{2}}=-(p-r)\{(p+h+s) \bar{F}(-z)-s\} \\
& \frac{\partial_{2} \Pi(p, q, r)}{\partial \beta_{2}}=-(p-r)^{2}(p+h+s) f(-z)<0
\end{aligned}
$$

Equation (57) proves the concavity of $\Pi(p, q, r)$. Equation (56) proves property (i) from the assumption that $p$ $+h>0$. The properties (ii) and (iii) are proved from property (i) and the fact that $\Pi(r, q, r)$ is constant for any value of $\beta_{2}$.

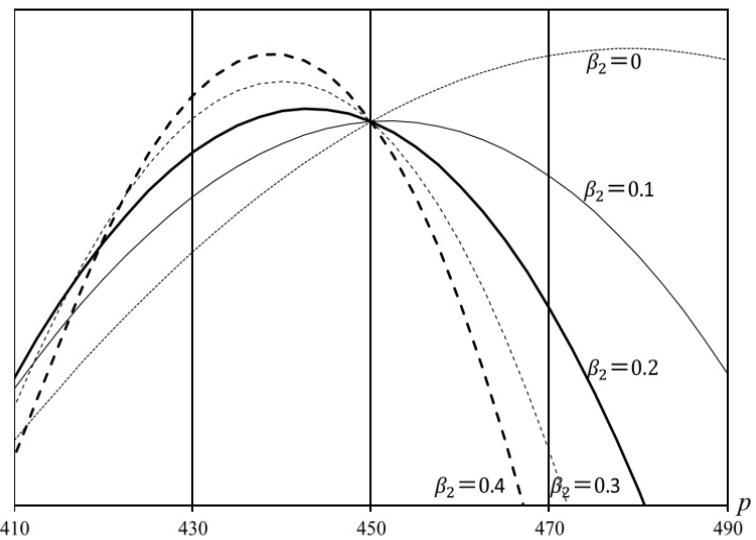

Figure 8. $\Pi(p, q, r)$ with Several Values of $\beta_{2}$ for LN Consumers where $r=450$.

Lemma 2 indicates that the contour of the expected profit function $\Pi(p, q, r)$ shown in Figure 8 which resembles that of $\pi_{\Lambda}(p, q, r)$ shown in Figure 4. Figure 9 depicts the fluctuation of $\Pi(p, q, r)$ with respect to $\beta_{2}$ for $p=410,430,450,470$, and 490 where $r=450$. The parameters in Figure 9 are set to the same values in Figure 8. In Figure 9, $\Pi(p, q, r)$ fluctuates as mentioned in property (i) of Lemma 2 except in the case $p=410$. The function $\Pi(p, q, r)$ with $p=410$ decreases for $\beta_{2}>0.2$, where Inequality (52) does not hold. 


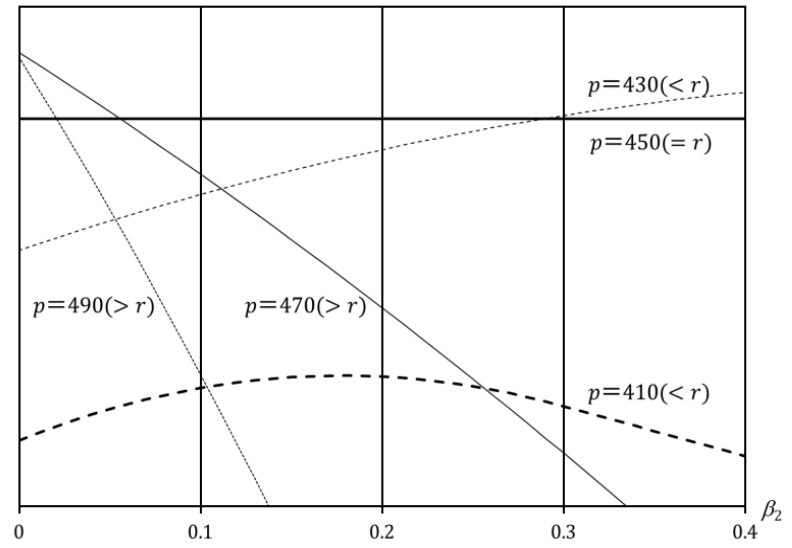

Figure 9. Fluctuation of $\Pi(p, q, r)$ with Respect to $\beta_{2}$ for LN Consumers where $r=450$.

\subsection{Optimal Pricing for Asymmetry Consumers}

In the case of $\beta_{2 G} \neq \beta_{2 L}$, if Inequality (52) holds, Lemma 2 shows that the expected profit function $\Pi(p$, $q, r)$ is expressed as

$$
\Pi(p, q, r)= \begin{cases}\min \left\{\Pi_{G}(p, q, r), \Pi_{L}(p, q, r)\right\} & \text { if } \beta_{2 G}<\beta_{2 L} \\ \max \left\{\Pi_{G}(p, q, r), \Pi_{L}(p, q, r)\right\} & \text { if } \beta_{2 G}>\beta_{2 L}\end{cases}
$$

where $\Pi_{G}(p, q, r)$ and $\Pi_{L}(p, q, r)$ are the $\Pi(p, q, r)$ with $\beta_{2}=\beta_{2 G}$ and $\beta_{2}=\beta_{2 L}$, respectively. The two functions $\Pi_{G}(p, q, r)$ and $\Pi_{L}(p, q, r)$ have a common point on $p=$ $r$. Figure 10 illustrates an example of the contour of $\Pi$ $(p, q, r)$ with several values of $\beta_{2 G}$. All of the functions $\Pi(p, q, r)$ are concave in Figure 10 . When $\beta_{2 G}=0$ or 0.05 , namely in the case of LA, the optimal price is equal to the reference price $r$, otherwise $\Pi_{G}(p, q, r)$ has the optimum.

Similarly to the deterministic case, let $\hat{p}_{G}(r)$ and $\hat{p}_{L}(r)$ be the prices to maximize $\Pi_{G}(p, q, r)$ and $\Pi_{L}(p$, $q, r)$, respectively. A theorem is proved as a procedure to derive the optimal price for asymmetry consumers in the stochastic case.

Theorem 4: When both the demand function $D(p, r)$ and the inventory level $Q$ are stochastic and consumers are LA or LS, the optimal price $p^{*}(q, r)$ which maximizes the expected profit $\Pi(p, q, r)$ is derived by Equations (59) and (60) along with Equation (31):

$$
\begin{aligned}
& p^{*}(q, r)=\arg \max _{p \in P_{2}^{*}} \Pi(p, q, r), \\
& P_{2}^{*}=\left\{\min \left[\max \left\{p, p_{L}\right\}, p_{H}\right] \mid p \in P_{1}^{*}\right\}
\end{aligned}
$$

\section{if Inequality (52) holds for $p=p_{L}$.}

Proof: Differentiating the left hand side of Inequality (52) with respect to $p$ shows that the left hand side monotonically increases with respect to $p$. Inequality (52) hence holds for all $p$ in the range $\left[p_{L}, p_{H}\right]$ if it holds for $p=p_{L}$. Under the situation, Lemma 2 classifies the shape of $\Pi(p, q, r)$ in the same way as the deterministic case shown in Figures 6 and 7 for LA and LS consumers, respectively. Consequently, the optimal price $p^{*}(q, r)$ is given by Equations (31), (59), and (60).

It is noticeable that Inequality (52) can be written as follows:

$$
\operatorname{Pr}(Q>D(p, r))>\frac{s}{p+h+s},
$$

since $\bar{F}(z)=\operatorname{Pr}(\varepsilon>z)$. Inequality (61) has the same form as the well-known critical fractile for newsvendor problems. It could be practical that the unit penalty $\operatorname{cost} s$ for an opportunity loss is recognized as zero for daily perishable products. Inequality (61) always holds when $s=0$.

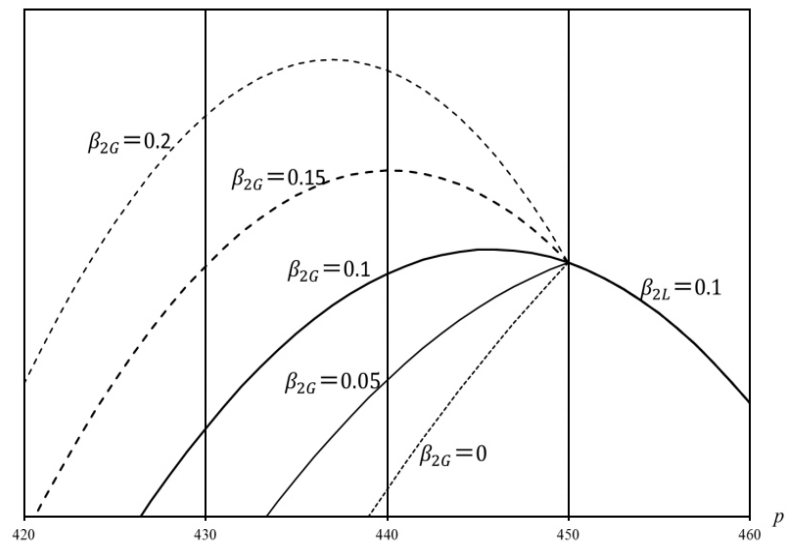

Figure 10. $\Pi(p, q, r)$ with Several Values of $\beta_{2 G}$ where $\beta_{2 L}=0.1$ and $r=450$.

\section{CONCLUSION}

In this paper, an optimal clearance pricing in a single period has been discussed analytically considering consumer's reference price effect. The profit function in deterministic case is concave if target consumers are LN or LA. For LS consumers, the function is concave or bimodal. In the stochastic case, if Inequality (52) holds for $p=p_{L}$, the contour of the expected profit function is similar to that of profit function in the deterministic case. The optimal price is obtained through the procedure proposed as Theorem 4 . The optimal clearing price depends especially on consumers' response, namely LN, LA, or LS.

The resulting theorems in this paper can be applied to the optimal clearance pricing in a multi-period case, which is the goal of our forthcoming study. The model in this paper can be modified to a combinatorial optimization, in which the firm determines the clearance price among several selectable prices, such as $10 \%$ off, $20 \%$ off, and $50 \%$ off. The modified model is more practical 
and the theorems in this paper could serve to reduce computational time to explore the optimal solutions.

\section{ACKNOWLEDGEMENT}

The authors gratefully acknowledge the constructive suggestions and helpful comments from anonymous referees. This work was partly supported by Grant-in-Aid for Scientific Research (C) No.22510147 and MEXT, Japan.

\section{REFERENCES}

Arrow, K., Harris, T., and Marschak, J. (1951), Optimal inventory policy, Econometrika, 19, 250-272.

Greenleaf, E. A. (1995), The Impact of Reference Price Effects on the Profitability of Price Promotions, Marketing Science, 14(1), 82-104.

Kahneman, D. and Tversky, A. (1979), Prospect theory:
An analysis of decision under risk, Econometrica, 47, 263-291.

Kalyanaram, G. and Winer, R. S. (1995), Empirical Generalization from Reference Price Research, Marketing Science, 14(3), G161-169.

Koide, T. and Sandoh, H. (2009), Reference Price Effect and Inventory Constraint on Optimal Pricing for Daily Perishable Products, Proceedings on the 2009 IEEE International Conference on Industrial Engineering and Engineering Management (IEEM), Hong Kong, 370-374.

Kopalle, P. K., Rao, A. G., and Assuncao, J. L. (1996), Asymmetric Reference Price Effects and Dynamic Pricing Policies, Marketing Science, 15(1), 60-85.

Petruzzi, N. C. and Dada, M. (1999), Pricing and the Newsvendor Problem: A Review with Extensions, Operations Research, 47(2), 183-194.

Popescu, I. and Wu, Y. (2007), Dynamic Pricing Strategies with Reference Effects, Operations Research, 55(3), 413-429. 